\title{
PACEM: a new concept for high avalanche-ion blocking
}

\author{
J.F.C.A. Veloso ${ }^{a, *}$, F.D. Amaro ${ }^{\mathrm{b}}$, C.D.R. Azevedo ${ }^{\mathrm{a}}$, J.M.F. dos Santos ${ }^{\mathrm{b}}$, A. Breskin ${ }^{\mathrm{c}}$, \\ A. Lyashenko ${ }^{\mathrm{c}}, \mathrm{R}$. Chechik ${ }^{\mathrm{c}}$ \\ ${ }^{a}$ Departmento de Fisica, Universidade de Aveiro, P-3810-193 Aveiro, Portugal \\ ${ }^{\mathrm{b}}$ Departmento de Fisica, Universidade de Coimbra, P-3004-516 Coimbra, Portugal \\ ${ }^{\mathrm{c}}$ Department of Particle Physics, The Weizmann Institute of Science, 76100 Rehovot, Israel
}

Available online 1 August 2007

\begin{abstract}
We present the Photon-Assisted Cascaded Electron Multiplier (PACEM) as a potential alternative for ion back-flow suppression in gaseous cascade electron multipliers. Using a Micro Hole and Strip Plate-Gas Electron Multiplier (MHSP-GEM) configuration, the number of ions flowing back to the scintillation region is about 1.5 ions per primary electron at an optical gain of 6.5 and a drift field of $0.1 \mathrm{kV} / \mathrm{cm}$, and about 10 ions per primary electron at an optical gain of 10 and a drift field of $0.5 \mathrm{kV} / \mathrm{cm}$. These allow reaching ion back-flow values close to $10^{-4}$ and $10^{-5}$ at typical operation conditions of TPCs and GPMs, respectively.

(C) 2007 Elsevier B.V. All rights reserved.
\end{abstract}

PACS: 29.40.Cs; 29.40.Gx; $85.60 . \mathrm{Gz}$

Keywords: Gaseous electron multipliers; MHSP; GEM; Gaseous photomultipliers; VUV scintillation; Photocathodes

\section{Introduction}

The positive ions produced in electron avalanches of gaseous detectors are responsible for secondary feedback effects, which limit the detector performance and the electron multiplication gain. In gaseous photomultipliers (GPMs), ions flowing back and impinging the photocathode (PC) induce its physical and chemical ageing and trigger secondary avalanches, which cause gain limitations and localization deterioration [1]. These effects are particularly important in visible light-sensitive GPMs due to the PC's high secondary emission probability. In time projection chambers (TPCs), ions flowing back to the conversion/drift region locally modify the electric field, resulting in dynamic track distortions [2]. This seriously affects the tracking properties of TPCs in highmultiplicity experiments, e.g. in relativistic heavy-ion physics applications.

\footnotetext{
${ }^{*}$ Corresponding author.

E-mail address: joao.veloso@ua.pt (J.F.C.A. Veloso).
}

The suppression of the ion back-flow (IBF) to the drift region and the $\mathrm{PC}$ has been realized to be a key issue for the detector's performance. IBF is defined as the fraction of ions reaching the $\mathrm{PC}$ or the drift region, relative to the total number of electrons collected in the multiplier's anode. For TPCs, this value should be of the order of $10^{-4}$ for gains of $10^{4}$ [2], while for GPMs it should stay below $10^{-5}$ for gains above $10^{5}[1]$. The IBF could be reduced at best to $10^{-3}$ and $10^{-4}$ in DC and in pulse-gate modes, respectively. The best ion-blocking performances so far were reached in cascaded GEMs and MHSPs [3,4], and in micromegas multipliers [5]. However, these IBF values are still too high for some applications, e.g. in TPCs and GPMs for the visiblespectral range.

In an attempt to further reduce the IBF, the PhotonAssisted Cascaded Electron Multiplier (PACEM) concept was introduced [6]. The PACEM uses the light produced in the electron avalanche in the first element of the cascade multiplier for signal amplification and transmission to the next cascade element, while a mesh placed in the transfer region between these elements, kept at a fixed voltage, is used to block both electrons and ions, (Fig. 1). This light is 


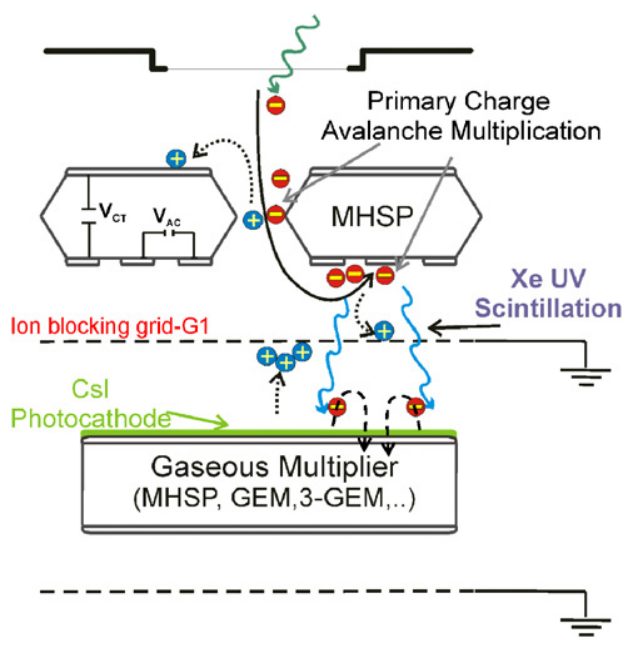

Fig. 1. The PACEM concept.

detected by a CsI-film evaporated on the second element upper surface, being the signal carried by the photoelectrons, which undergo amplification in the subsequent elements of the cascade. This way, signal transmission is obtained by optical coupling, while the charge is decoupled. Under these conditions, regardless of the total gain of the cascaded multiplier, only ions originating in the first element will flow back to the initial drift region, or to the top surface of the first multiplier.

With a proper design of the photon-mediated stage, the number of photoelectrons induced on the PC at the second stage largely exceeds the number of primary electrons in the drift region, resulting in what we define as optical gain.

This opto-coupling technique may be applied between the first two stages of GPMs' and TPCs' readout elements as a means for reducing the IBF, provided that ways and conditions are found to reduce the first stage's IBF to a minimum acceptable value. It can be applied in many different cascaded-multiplier configurations. The first element can be, for instance, a wire chamber, a parallelgrid multiplier or a GEM. Preferably, it should be an element that provides maximal light yield for a given gas gain, as to generate the minimal number of ions per event. The cascade may comprise elements of different types; the number of elements of each type will be chosen according to the desirable total gain. This technique is only effective in gases presenting high scintillation efficiency, like noble gases and $\mathrm{CF}_{4}$. Almost simultaneously, its non-functionality for mixtures with organic gases has been demonstrated [7].

In this work, we investigate the capability of the PACEM to suppress IBF in electron multiplier cascades operating in a xenon atmosphere. The optical gain of the PACEM, i.e. the number of photoelectrons collected per primary electron, and the relative IBF, i.e. the number of ions per primary electron (IBF/pe) flowing back to the drift region, are studied as a function of the voltages applied to the multiplier elements.

\section{Experimental set-up}

The PACEM used in this work is depicted schematically in Fig. 2.

The first element of the cascade is a MHSP [8]. The two consecutive amplification stages of the MHSP, a GEM-like hole avalanche stage followed by a MSGC-type avalanche stage at the MHSP bottom surface, provide higher scintillation yields when compared to a GEM [6], while blocking efficiently the ions produced in the charge avalanche of the second stage. As discussed in Ref. [9], depending on the induction field between the MHSP and the wire mesh, only $\sim 20 \%$ of the final-avalanche ions may drift back through the MHSP holes. Therefore, the MHSP bias can provide low charge gain in the holes, while pushing the gain in the anode strips in order to maintain high scintillation yields with reduced IBF.

The second element is a GEM coated with a $500 \mathrm{~nm}$ thick reflective CsI-film to readout the scintillation produced in the first element. A null electric field is established in the region between the wire mesh and the GEM for maximum photoelectron transmission efficiency through the GEM [10].

The two elements are optically coupled, within the same gas volume, through a grounded charge-blocking meshelectrode (stainless steel wires with $80 \mu \mathrm{m}$ diameter and $900 \mu \mathrm{m}$ spacing). The primary charge was produced using a photon-electron converter based on a CsI-semitransparent photocathode (ST-PC), deposited on a quartz window placed a few millimetres above the MHSP, and irradiated by a $\mathrm{Hg}$ lamp. The primary electrons are focussed into the holes of the MHSP and create an avalanche within the holes; a second avalanche occurs at the anode strips, where all electrons are collected [8]. Most of the avalanche ions are collected in the grid mesh and in the MHSP anodes and only $\sim 20 \%$ will flow back into the holes. Avalanchegenerated photons induce photoelectrons from the CsIreflective photocathode (R-PC) deposited on the top surface of the GEM; these are focused into the GEM

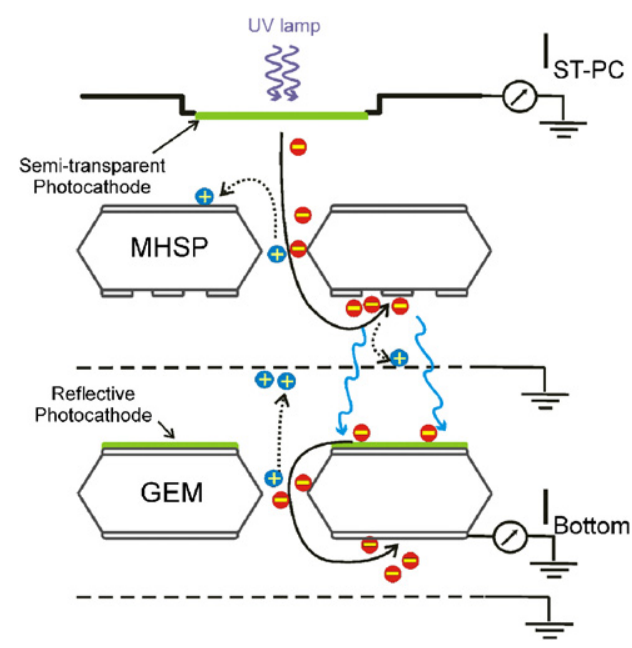

Fig. 2. Schematic view of the PACEM setup used in this work. 
holes and create the final avalanche in these holes. The present studies were performed operating the PACEM in current mode.

\section{Experimental results and discussion}

We have measured, simultaneously, the optical gain and the relative IBF, IBF/pe, as a function of the voltages on the MHSP electrodes: the voltage difference applied to the holes, $V_{\mathrm{CT}}$, and the voltage difference applied between anode and cathode strips, $V_{\mathrm{AC}}$. For simplicity, in these measurements, the photoelectrons emitted from the R-PC of the second element were collected in the charge-blocking mesh-electrode, by applying an electric field of $1 \mathrm{kV} / \mathrm{cm}$ to the region above the GEM, and maintaining the $V_{\mathrm{GEM}}$ voltage at ground level. These conditions ensure the full photoelectron collection at the mesh.

The optical gain is given by

$G_{\mathrm{opt}}=\left(I_{\mathrm{R}-\mathrm{PC}}-I_{\mathrm{peR}}\right) / I_{\mathrm{pe}}$.

$I_{\mathrm{R}-\mathrm{PC}}$ is the current measured at the R-PC. $I_{\mathrm{peR}}$ denotes the photoelectron current due to direct exposure of the RPC to the UV photons emitted by the $\mathrm{Hg}$ lamp that pass through the MHSP holes. $I_{\mathrm{pe}}$ is the primary photoelectron current on the ST-PC, resulting from the absorption of VUV photons emitted by the Hg lamp. $I_{\mathrm{pe}}$ and $I_{\mathrm{peR}}$ are measured for null electric fields in the MHSP, i.e. $V_{\mathrm{CT}}=V_{\mathrm{AC}}=0 \mathrm{~V}$. For the optical gain measurements, the drift electric field, above the MHSP, was kept at $0.3 \mathrm{kV} / \mathrm{cm}$.

The optical gain as a function of the MHSP electrode voltages is presented in Fig. 3. The left part of the upper curve was obtained increasing $V_{\mathrm{CT}}$ from 270 to $450 \mathrm{~V}$, while keeping $V_{\mathrm{AC}}=0 \mathrm{~V}$, i.e. operating the MHSP in GEM mode. A maximum optical gain of 1 was obtained for the MHSP operation in GEM mode. This shows that the GEM is not suitable to be used as the first multiplier element of the PACEM. Additional charge and scintillation is achieved

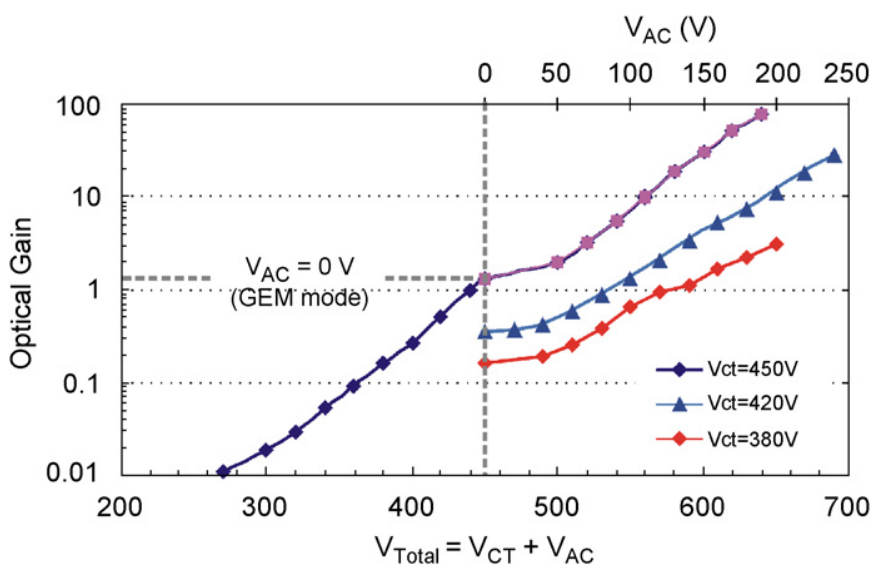

Fig. 3. PACEM optical gain as a function of the MHSP electrode voltages. The left part of the upper curve was obtained keeping $V_{\mathrm{AC}}=0 \mathrm{~V}$. The right part of the upper curve and the lower curves were obtained by increasing $V_{\mathrm{AC}}$ while keeping $V_{\mathrm{CT}}$ constant. The upper curve can be read in relation to the lower as well as to the upper scale of the abscissa. The two lower curves are only related to the upper scale. in the MHSP by biasing the anode strips, increasing $V_{\mathrm{AC}}$. A strong increase of the optical gain to values of about 80 was obtained (see Fig. 3). The relative IBF is given by

$$
\mathrm{IBF} / \mathrm{pe}=\left(I_{\mathrm{ST}-\mathrm{PC}}-I_{\mathrm{pe}}\right) / I_{\mathrm{pe}} .
$$

$I_{\mathrm{ST}-\mathrm{PC}}$ is the current measured at the ST-PC and $I_{\mathrm{pe}}$ the primary photoelectron current in the ST-PC, as measured above. In Fig. 4 we depict the relative IBF as a function of the optical gain for different MHSP electrode voltages and for drift electric fields of 0.1 and $0.5 \mathrm{kV} / \mathrm{cm}$. The total number of ions flowing back to the drift region is $1.5 \mathrm{ions} /$ pe for an optical gain of 6.5 and a drift field of $0.1 \mathrm{kV} / \mathrm{cm}$, see Fig. 4a. For a drift field of $0.5 \mathrm{kV} / \mathrm{cm}$, the relative IBF is about 10 ions/pe for an optical gain of about 10 , see Fig. 4b. These numbers demonstrate that it is feasible to achieve an IBF close to $10^{-4}$ at a drift field of $0.1 \mathrm{kV} / \mathrm{cm}$, and $10^{-5}$ at a drift field of $0.5 \mathrm{kV} / \mathrm{cm}$, for total multiplier gains of $10^{4}$ and $10^{6}$, respectively.

To demonstrate that the second element, as well as any subsequent ones, do not contribute to the IBF of the PACEM, the relative IBF and the total PACEM gain were measured as a function of $V_{\mathrm{GEM}}$, for a null electric field in the region above the GEM, a drift electric field of $0.3 \mathrm{kV} / \mathrm{cm}$ and constant $V_{\mathrm{CT}}$ and $V_{\mathrm{AC}}$ voltages in the MHSP. For this study, we measured the current at the ST-PC as well as the current at the GEM bottom. Increasing the GEM voltage

a

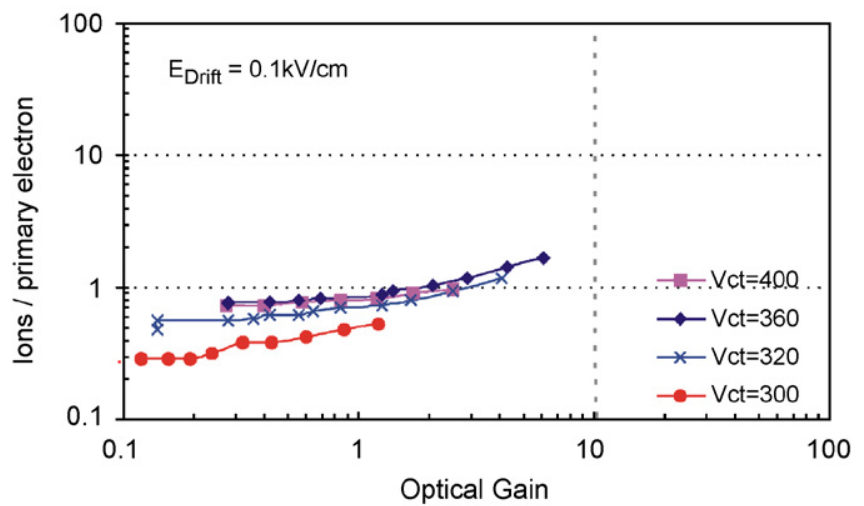

b

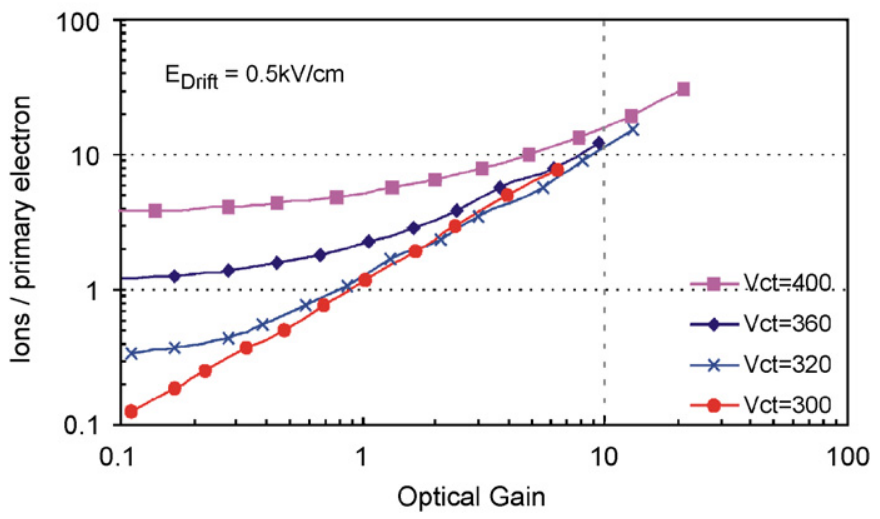

Fig. 4. Relative IBF as a function of the PACEM optical gain for drift electric fields of (a) $0.1 \mathrm{kV} \mathrm{cm}$ and (b) $0.5 \mathrm{kV} \mathrm{cm}$ and for different $V_{\mathrm{CT}}$ voltages in the MHSP. 
the total gain increases, but the current on the ST-PC due to the IBF remains constant, demonstrating the $100 \%$ efficiency of the charge-blocking mesh.

\section{Conclusion}

We have investigated the potential of the PACEM concept to suppress ion back-flow (IBF), when used in electron multiplier cascades operating in a xenon atmosphere. We demonstrated that the IBF depends only on the bias applied to the first element, being independent of the elements placed below the charge-blocking mesh and of the cascade total gain. Using a MHSP as the first element of a PACEM, it is possible to obtain IBFs around $10^{-4}$ for gains of $10^{4}$, and $10^{-5}$ for gains of $10^{6}$, for drift electric fields of 0.1 and $0.5 \mathrm{kV} / \mathrm{cm}$, respectively, i.e. the typical operation conditions of TPCs and GPMs. Light gains of about 100 are possible to reach in the PACEM, though at cost of an increased IBF.

In future work we will investigate the performance of the PACEM operating in $\mathrm{CF}_{4}$, a more interesting gas in highenergy instrumentation. Although the UV scintillation yield of $\mathrm{CF}_{4}$ is about 10 times less than in xenon, the photoelectron collection efficiency in $\mathrm{CF}_{4}$ is more than a factor of three higher than in xenon. Therefore, the potential of the PACEM concept in reducing the IBF in $\mathrm{CF}_{4}$ atmosphere may approach that obtained in a xenon atmosphere.

\section{Acknowledgements}

This work was supported in part by Project POCI/FP/ 63962/2005 through FEDER and FCT (Lisbon) programs, by the MINERVA Foundation and by the Israel Science Foundation project 402/05. A. Breskin is the W.P. Reuther Professor of Research in peaceful use of atomic energy.

\section{References}

[1] A. Breskin, T. Boutboul, A. Buzulutskov, R. Chechik, E. Shefer, G. Garty, B.K. Singh, Nucl. Instru. Meth. A 442 (2000) 58.

[2] F. Sauli, GEM readout of the time projection Chamber, CERN-EP TA1 Internal Report, July 29, 1999.

[3] A. Breskin, D. Mörmann, A. Lyashenko, R. Chechik, F.D. Amaro, J.M. Maia, J.F.C.A. Veloso, J.M.F. dos Santos, Nucl. Instr. and Meth. A 553 (2005) 46 and references therein.

[4] A.V. Lyashenko, A. Breskin, R. Chechik, J.F.C.A. Veloso, J.M.F. dos Santos, F.D. Amaro, J. Inst. 1 (2006) P10004.

[5] P. Colas, I. Giomataris, V. Lepeltier, Nucl. Instr. Meth. A 535 (2004) 226.

[6] J.F.C.A. Veloso, F.D. Amaro, J.M.F. dos Santos, A. Breskin, A.V. Lyashenko, R. Chechik, J. Inst. 1 (2006) P08003.

[7] A. Buzulutskov, A. Bondar, J. Inst. 1 (2006) P08006.

[8] F.D. Amaro, J.F.C.A. Veloso, A. Breskin, R. Chechik, J.M.F. dos Santos, J. Inst. 1 (2006) P0400.

[9] J.M. Maia, D. Mörmann, A. Breskin, R. Chechik, J.F.C.A. Veloso, J.M.F. dos Santos, Nucl. Instr. and Meth. A 523 (2004) 334.

[10] D. Mörmann, A. Breskin, R. Chechik, C. Shalem, Nucl. Instr. and Meth. A 530 (2004) 258. 